Review Article

\section{A Perspective on therapeutic potential of weeds}

\author{
Vikrant Arya ${ }^{1 *}$ and Ranjeet Kaur Parmar ${ }^{2}$ \\ ${ }^{1}$ Assistant Professor, Government College of Pharmacy, Rohru, Shimla, Himachal Pradesh, India \\ ${ }^{2}$ Home Maker, Pranav Kuteer, Jayanti Vihar, Kangra, Himachal Pradesh, India
}

\section{Abstract}

Nature gives us a diverse plethora of floral wealth. Weeds have been recognized as invasive plant by most of scholars in today's world with extraordinary travel history. They are considered to be noxious for adjoining plant species and also as economic hazard. Weeds inhabited in almost entire biomes and have capability to survive in harsh conditions of environment thereby become source of inspiration for finding novel phytoconstituents. Weeds play a significant role in absorbing harmful micro pollutants that are affecting ecosystem adversely. There are so many examples like canna lily, bladder wort, coltsfoot, giant buttercup etc. playing crucial part in sustaining environment. Different isolation and characterization approaches like high pressure liquid chromatography, gas chromatography, ion exchange chromatography, nuclear magnetic resonance, mass spectroscopy etc. have also been fetched for obtaining novel constituents from weeds. The main aim of this review is to analyze the therapeutic potential of weeds established in New Zealand and effort to unfold the wide scope of its applications in biological sciences. Upon exploration of various authorized databases available it has been found that weeds not only are the reservoir of complex phytoconstituents exhibiting diverse array of pharmacological activities but also provide potential role in environment phytoremediation. Phytoconstituents reported in weeds have immense potential as a drug targets for different pathological conditions. This review focuses on the literature of therapeutic potential of weeds established in New Zealand and tried to unveil the hidden side of these unwanted plants called weeds.

\section{More Information}

*Address for Correspondence: Vikrant Arya Assistant Professor, Government College of Pharmacy, Rohru, Shimla, Himachal Pradesh-171207, India, Tel: 8628998699; Email: arya.vikrant30@gmail.com

\section{Submitted: 04 June 2020 \\ Approved: 17 June 2020 \\ Published: 18 June 2020}

How to cite this article: Arya V, Parmar RK. A Perspective on therapeutic potential of weeds. J Plant Sci Phytopathol. 2020; 4: 042-054.

DOI: 10.29328/journal.jpsp.1001050

\section{ORCiD: orcid.org/0000-0002-6808-1754}

Copyright: @ 2020 Arya V, et al. This is an open access article distributed under the Creative Commons Attribution License, which permits unrestricted use, distribution, and reproduction in any medium, provided the original work is properly cited.

Keywords: New Zealand; Phytoconstituents; Phytoremediation; Weeds

(D) Check for updates

OPEN ACCESS

\section{Introduction}

'Horse Hoeing Husbandry' named famous writing by Jethro Tull (1731) mentioned first time the word 'weed' [1]. Weeds may be considered as plants whose abundance must be over or above a specific level can cause major environmental concern [2]. Aldrich and Kremer, 1997 defined weed as a part of dynamic ecosystem [3]. Plant originated in natural environment and, in response to imposed or natural environments, evolved, and continues to do so, as an interfering associate with crops and activities. Weeds may interfere with the utilization of land and water resources thereby adversely affect human welfare [4]. According to Ancient Indian Literature earth is blessed with diverse flora and every existing plant has their own importance. Some plants are considered unwanted but they may have beneficial properties. Scholars are trying hard to explore the hidden potential of such unwanted plants [5]. Weeds have interactions with other organisms and some of these interactions can have direct effects on the functioning of agro-ecosystem [6]. They serve as an indirect resource for predatory species [7] and it could alternative food sources for organisms that play prominent role in insect control [8] Weeds have a unique travel history. Clinton L. Evans in his book 'The war on weeds in the prairie west- An Environmental History' mentioned about travelling of weeds in ships, railways, automobiles from one country to another as food contaminants, animal feed, farm implements etc. during trade [9]. Weeds are firmly distributed and established all over New Zealand. Authors Ian Popay, Paul Champion and Trevor James in their book 'An Illustrated Guide to Common Weeds of New Zealand' (edition $3^{\text {rd }}$ ) published by New Zealand Plant Protection Society in 2010 mentioned the detailed description of around 380 weed species established in New Zealand [10]. Different scientific databases/ information resources (governmental, private, universities, initiatives, organizations etc.) of New Zealand extensively explored over a year as mentioned in table 1 to obtain data pertaining to weeds prevalent within geographical boundaries of New Zealand. After obtaining desired data of different weeds, a literature search was performed using the keyword "Name of weed (e.g. Aristea ecklonii) Pharmacology", "New Zealand plants", "weed pharmacology", "therapeutic weed" individually or all together in different scientific databases of Scopus, Web of Science and Pubmed to obtain therapeutic potential of weeds. Celastrus orbiculatus (Climbing spindle berry) [59], Robinia pseudoacacia (False acacia) [63], Daphne laureola 
Table 1: Description of scientific databases/ information resources of New Zealand for weed identification.

\begin{tabular}{|c|c|c|c|c|}
\hline \multicolumn{3}{|c|}{ PRIMARY INFORMATION SOURCES } & \multicolumn{2}{|r|}{ SECONDARY INFORMATION SOURCES* } \\
\hline Source name & Source type & Authors & Web address & Database/information resource \\
\hline An encyclopedia of New Zealand, 1966 & Encyclopedia & McLintock AH & http://www.agpest.co.nz & $\begin{array}{c}\text { AgPest: It is an open access tool available for New Zealand } \\
\text { farmers and agricultural professionals containing information } \\
\text { about weeds, pest identification, their biology, impact and } \\
\text { management }\end{array}$ \\
\hline Common weeds in New Zealand, 1976 & Book & $\begin{array}{l}\text { Parham BEV, } \\
\text { Healy AJ }\end{array}$ & $\begin{array}{l}\text { http://www.agriculture.vic. } \\
\text { gov.au }\end{array}$ & $\begin{array}{l}\text { Agriculture victoria : Platform is used to promote agriculture } \\
\text { industry in New Zealand and encompass information related to } \\
\text { weeds and plant protection }\end{array}$ \\
\hline $\begin{array}{l}\text { Weeds in New Zealand protected } \\
\text { natural areas: A review for the } \\
\text { Department of Conservation, } 1990\end{array}$ & Book & $\begin{array}{l}\text { Williams PA, } \\
\text { Timmins SM }\end{array}$ & $\begin{array}{l}\text { http://www.cropscience. } \\
\text { bayer.co.nz }\end{array}$ & $\begin{array}{l}\text { Bayer crop science: It is one of the major information providers } \\
\text { of crop protection products }\end{array}$ \\
\hline $\begin{array}{l}\text { Problem weeds on New Zealand } \\
\text { islands, } 1997\end{array}$ & $\begin{array}{c}\text { Book } \\
\text { ISBN 0-478-01885-1 }\end{array}$ & Atkinson IAE & http://www.gw.govt.nz & $\begin{array}{l}\text { Greater wellington university: It is a local government body in } \\
\text { New Zealand represented by regional and territorial councils }\end{array}$ \\
\hline $\begin{array}{l}\text { New Zealand Journal of Agricultural } \\
\text { Research, 50(2), } 2007\end{array}$ & Journal & $\begin{array}{c}\text { Bourdot GW, } \\
\text { Fowler SV, } \\
\text { Edwards GR, } \\
\text { Kriticos DJ, } \\
\text { Kean JM, } \\
\text { Rahman A, } \\
\text { Parsons AJ }\end{array}$ & http://www.learnz.org.nzs & $\begin{array}{c}\text { Learnz: It is a initiative of free virtual field trips that help } \\
\text { students to acquire inaccessible knowledge regarding various } \\
\text { agricultural activities }\end{array}$ \\
\hline $\begin{array}{l}\text { Consolidated list of environmental } \\
\text { weeds in New Zealand, } 2008\end{array}$ & $\begin{array}{l}\text { Journal } \\
\text { ISBN 978-0-478- } \\
14412-3\end{array}$ & Howell C & http://www.massey.ac.nz & $\begin{array}{c}\text { Massey university: In Massey University, College of Sciences } \\
\text { prepared a database dedicated to provide information } \\
\text { regarding weeds in New Zealand }\end{array}$ \\
\hline $\begin{array}{l}\text { New Zealand Journal of Ecology, } 33 \\
\text { (2), } 2009\end{array}$ & Journal & $\begin{array}{l}\text { Sullivan JJ, } \\
\text { Williams PA, } \\
\text { Timmins SM, } \\
\text { Smale MC }\end{array}$ & http://www.mpi.govt.nz & $\begin{array}{c}\text { Ministry for primary industries: The Ministry for Primary } \\
\text { Industries is dedicated to improving agriculture productivity, } \\
\text { food safety, increasing sustainability and reducing biological } \\
\text { risk }\end{array}$ \\
\hline $\begin{array}{l}\text { An illustrated guide to common weeds } \\
\text { of New Zealand, } 2010\end{array}$ & Book & $\begin{array}{l}\text { Popay } \mathrm{I}, \\
\text { Champion } \mathrm{P} \text {, } \\
\text { James } \mathrm{T}\end{array}$ & http://www.nzpcn.org.nz & $\begin{array}{l}\text { New Zealand plant conservation network: This network system } \\
\text { is framed to conserve the floral wealth of New Zealand }\end{array}$ \\
\hline $\begin{array}{l}\text { New Zealand Journal of Ecology, 39(1), } \\
\qquad 2015\end{array}$ & Journal & $\begin{array}{l}\text { McAlpine KG, } \\
\text { Lamoureaux } \\
\text { SL, } \\
\text { Westbrooke I }\end{array}$ & $\begin{array}{l}\text { http://www.ourbigbackyard. } \\
\text { nz }\end{array}$ & $\begin{array}{c}\text { Our big backyard: This aims to restore, create and maintain } \\
\text { healthy habitats of New Zealand }\end{array}$ \\
\hline Agronomy, 9, 2019 & Journal & $\begin{array}{l}\text { Ghanizadeh } \mathrm{H}, \\
\text { Harrington } \mathrm{KC}\end{array}$ & $\begin{array}{l}\text { http://www.waikoregion. } \\
\text { govt.nz }\end{array}$ & $\begin{array}{l}\text { Waikato: This local government body works for maintaining } \\
\text { agriculture resources and sustainability to ensure strong } \\
\text { economy }\end{array}$ \\
\hline $\begin{array}{l}\text { Climate change risk assessment for } \\
\text { terrestrial species and ecosystems in } \\
\text { the Auckland region. Auckland Council, } \\
2019\end{array}$ & $\begin{array}{l}\text { Technical report } \\
\text { ISBN 978-1-98- } \\
\quad 858966-4\end{array}$ & $\begin{array}{l}\text { Bishop C, } \\
\text { Landers TJ }\end{array}$ & $\begin{array}{l}\text { http://www.weedbusters. } \\
\text { org.nz }\end{array}$ & $\begin{array}{l}\text { Weedbusters: Programme facilitates to eradicate weeds in } \\
\qquad \text { New Zealand }\end{array}$ \\
\hline
\end{tabular}

(Green daphne laurel) [66], Glaucium flavum (Horned poppy) [70], Senecio latifolius (Pink ragwort) [80], Solanum nigrum (Black night shade) [86] have potent anticancer activities. Aristea ecklonii (Aristea) [50], Alocasia brisbanensis (Elephant ear) [62], Lycopus europaeus (Gypsywort) [68] exhibited antimicrobial activities. Pseudosasa japonica (Arrow bamboo) [51], Sambucus nigra (Elder) [61], Equisetum arvense (Field horsetail) [65] showed antioxidant effect. Hedera helix (Ivy) [72], Nymphaea Mexicana (Mexican water lily) [75], Persicaria hydropiper (Water pepper) [106] showed anti-inflammatory properties. Weeds like Zantedeschia aethiopica (Arum lily) [112], Utricularia gibba (Bladderwort) [113], Canna indica (Canna lilly) [114], Tussilago farfara (Coltsfoot) [115], Egeria densa (Eregia) [116], Ranunculus acris (Giant buttercup) [117], Cytisus scoparius (Broom) [118], Poa annua (Annual poa) have prominent role in biomonitoring of heavy metals in multiple environments [119].

\section{Chemical profile of weeds established in New Zealand}

Weeds established in New Zealand encompass wide array of therapeutic phytoconstituents. Weeds serve as biosynthetic factory for synthesis of phytochemicals. They are sources of rich medicinal wealth which includes primary metabolites (polysaccharides) and secondary metabolites (alkaloids, flavonoids, glycosides, tannins, volatile oils etc.). They are the potential sources of complex phytoconstituents. Selaginella kraussiana (African club moss) [11], Lonicera japonica (Japnese honeysuckle) [32], Eriobotrya japonica (Loquat) [35] and Anredera cordifolia (Mignonette vine) [38] contains polysaccharides. Alternanthera philoxeroides (Alligator weed) [13] and Rhamnus alaternus (Evergreen buckthorn) [26] contains anthraquinone glycosides. Lamium galeobdolon (Artillery plant) [14] and Heracleum mantegazzianum (Giant hogweed) [27] contains appreciable amount of volatile oil. Modern spectroscopic methods have been explored for structural elucidation of bioactive constituents present in weeds. LC-MS has been used for quantitative detection of xyloglucan oligosaccharide in Selaginella kraussiana [11], betulonic acid in Alnus glutinosa (Black alder) [12], jasmonic acid in Drosera capensis (Cape sundew) [20], flavonoids in Gunnera tinctoria (Chilean rhubarb) [24], pyrrolizidine alkaloid esters in Gymnocoronis spilanthoides (Senegal tea) 
[42]. NMR employed for characterization of compounds present in Fraxinus excelsior (Ash) [15], Berberis glaucocarpa (Barberry) [17], Ligustrum sinense (Chinese privet) [25], Rhamnus alaternus [26], Cestrum parqui (Green cestrum) [30], Ranunculus sardous (Hairy buttercup) [49]. Detailed summary of chemical compounds isolated from weeds established in New Zealand indicated in table 2.

\section{Therapeutic potential of weeds established in New Zealand}

Weeds have been explored for diverse pharmacological actions like anti cancer, anti microbial, anti-inflammatory, antioxidant, antiviral etc. as mentioned in table 3 and figure 1.

Anticancer weeds: Some important cytotoxic weeds

Table 2: A summary of compounds isolated from weeds established in New Zealand.

\begin{tabular}{|c|c|c|c|c|c|}
\hline Common name & Botanical name & Native of & Compound reported & Analytical approach adopted & References \\
\hline African club moss & $\begin{array}{l}\text { Selaginella kraussiana } \\
\text { Selaginellaceae }\end{array}$ & Africa & Xyloglucan oligosaccharide & $\begin{array}{l}\text { Matrix assisted laser desorption ionization } \\
\text { time of flight (MS), high performance anion } \\
\text { exchange chromatography }\end{array}$ & [11] \\
\hline Black alder & $\begin{array}{c}\text { Alnus glutinosa } \\
\text { Betulaceae }\end{array}$ & Eurasia, Africa & Betulin, betulinic acid, betulonic acid, lupeol & $\begin{array}{l}\text { Desorption atmospheric pressure } \\
\text { photoionization (MS) }\end{array}$ & [12] \\
\hline Alligator weed & $\begin{array}{l}\text { Alternanthera } \\
\text { philoxeroides } \\
\text { Amaranthaceae }\end{array}$ & South America & Anthraquinone glycosides & Spectral analysis & [13] \\
\hline Artillery plant & $\begin{array}{c}\text { Lamium galeobdolon } \\
\text { Lamiaceae }\end{array}$ & Europe, Asia & Volatile compounds & GC-MS & [14] \\
\hline Ash & $\begin{array}{l}\text { Fraxinus excelsior } \\
\text { Oleaceae }\end{array}$ & Europe, Asia, Africa & Nodulisporiviridin M & ID, 2D 1H \& 13C NMR & [15] \\
\hline Asiatic knotweed & $\begin{array}{l}\text { Fallopia japonica } \\
\text { Polygonaceae }\end{array}$ & Asia & Carotenoid & HPTLC, HPLC-MS & [16] \\
\hline Barberry & $\begin{array}{l}\text { Berberis glaucocarpa } \\
\text { Berberidaceae }\end{array}$ & Himalayas & $\begin{array}{l}\text { Bisbenzylisoquinoline alkaloid, } \\
\text { oxyacanthine }\end{array}$ & 1D, 2D NMR & {$[17]$} \\
\hline Blackberry & $\begin{array}{l}\text { Rubus fruticosus } \\
\text { Rosaceae }\end{array}$ & $\begin{array}{l}\text { North temperate } \\
\text { regions }\end{array}$ & Polyunsaturated fatty acids & Supercritical carbon dioxide method & [18] \\
\hline Boxthorn & $\begin{array}{l}\text { Lycium ferocissimum } \\
\text { Solanaceae }\end{array}$ & South Africa & Betaine & Fast atom bombardment mass spectroscopy & [19] \\
\hline Cape sundew & $\begin{array}{l}\text { Drosera capensis } \\
\text { Droseraceae }\end{array}$ & South Africa & Jasmonic acid & LC-MS/MS & {$[20]$} \\
\hline Castor oil & $\begin{array}{l}\text { Ricinus communis } \\
\text { Euphorbiaceae }\end{array}$ & Africa, Eurasia & Ricin & Spectral analysis & [21] \\
\hline Century plant & $\begin{array}{c}\text { Agave americana } \\
\text { Agavaceae }\end{array}$ & Mexico & Fructans & Thermogravimetric analysis & [22] \\
\hline Cherry laurel & $\begin{array}{c}\text { Prunus laurocerasus } \\
\text { Rosaceae }\end{array}$ & South East Europe & $\begin{array}{l}\text { Cyanogenetic glycosides, benzoic acid } \\
\text { derivative }\end{array}$ & LC-ESIMS & [23] \\
\hline Chilean rhubarb & $\begin{array}{l}\text { Gunnera tinctoria } \\
\text { Gunneraceae }\end{array}$ & South America & Flavonoids & HPLC-MS/MS & [24] \\
\hline Chinese privet & $\begin{array}{l}\text { Ligustrum sinense } \\
\text { Oleaceae }\end{array}$ & China & $\begin{array}{l}\text { 10-hydroxyl-oleuropein, 3-O-alpha-L- } \\
\text { rhamnopyranosyl-kaempherol-7-O-beta-D- } \\
\text { glucopyranoside }\end{array}$ & 1D, 2D NMR & [25] \\
\hline $\begin{array}{l}\text { Evergreen } \\
\text { buckthorn }\end{array}$ & $\begin{array}{l}\text { Rhamnus alaternus } \\
\text { Rhamnaceae }\end{array}$ & $\begin{array}{l}\text { Mediterranean } \\
\text { region }\end{array}$ & Anthraquinone glycosides & $\begin{array}{l}\text { 1D, 2D NMR, } \\
\text { FAB-MS }\end{array}$ & [26] \\
\hline Giant hogweed & $\begin{array}{l}\text { Heracleum } \\
\text { mantegazzianum } \\
\text { Apiaceae }\end{array}$ & Eurasia & Essential oil & GC-MS & [27] \\
\hline Giant knotweed & $\begin{array}{l}\text { Fallopia sachalinensis } \\
\text { Polygonaceae }\end{array}$ & Asia & Olymeric procyanidins, flavones, flavonoids & GC-MS & [28] \\
\hline Giant reed & $\begin{array}{l}\text { Arundo donax } \\
\text { Gramineae }\end{array}$ & Eurasia & Bis-indole alkaloid, phenylpropanoid & Spectral analysis & [29] \\
\hline Green cestrum & $\begin{array}{l}\text { Cestrum parqui } \\
\text { Solanaceae }\end{array}$ & Chile, Peru & Saponin & $1 \mathrm{H}, 13 \mathrm{C} N \mathrm{NMR}$ & [30] \\
\hline Heather & $\begin{array}{l}\text { Calluna vulgaris } \\
\text { Ericaceae }\end{array}$ & Europe & Catechin, epicatechin & HPLC-DAD-ESI/MS & [31] \\
\hline $\begin{array}{c}\text { Japnese } \\
\text { honeysuckle }\end{array}$ & $\begin{array}{l}\text { Lonicera japonica } \\
\text { Caprifoliaceae }\end{array}$ & Japan & Polysaccharides & HPLC, FTIR & [32] \\
\hline Khasia berry & $\begin{array}{l}\text { Cotoneaster simonsii } \\
\text { Rosaceae }\end{array}$ & China & Tocopherols & Spectral analysis & [33] \\
\hline Kudzu vine & $\begin{array}{l}\text { Pueraria lobata } \\
\text { Fabaceae }\end{array}$ & Japan & Lobatamunsolides $\mathrm{A}-\mathrm{C}$, norlignans & LC-MS & [34] \\
\hline Loquat & $\begin{array}{l}\text { Eriobotrya japonica } \\
\text { Rosaceae }\end{array}$ & China, Japan & Polysaccharides & UMAE & [35] \\
\hline $\begin{array}{l}\text { Manchurian rice } \\
\text { grass }\end{array}$ & $\begin{array}{c}\text { Zizania latifolia } \\
\text { Poaceae }\end{array}$ & China & Proanthocyanidins & UAE & [36] \\
\hline Mexican devil & $\begin{array}{c}\text { Ageratina adenophora } \\
\text { Asteraceae }\end{array}$ & South America & Thymol derivatives & 1H NMR, HR-ESI-MS, IR & {$[37]$} \\
\hline
\end{tabular}




\begin{tabular}{|c|c|c|c|c|c|}
\hline Mignonette vine & $\begin{array}{l}\text { Anredera cordifolia } \\
\text { Basellaceae }\end{array}$ & South America & Water soluble polysaccharides & UV, FTIR & [38] \\
\hline Moth plant & $\begin{array}{l}\text { Araujia hortorum } \\
\text { Asclepiadaceae }\end{array}$ & Brazil, Argentina & Protease (araujiain) & $\begin{array}{l}\text { Ultracentrifugation, ion exchange } \\
\text { chromatography, MS }\end{array}$ & [39] \\
\hline Mysore thorn & $\begin{array}{c}\text { Caesalpinia decapetala } \\
\text { Fabaceae }\end{array}$ & Asia & Cassane type furanoditerpenoids & HPLC, 1D NMR, 2D NMR, HRESIFTMS & [40] \\
\hline Plectranthus & $\begin{array}{c}\text { Plectranthus ciliates } \\
\text { Lamiaceae }\end{array}$ & South Africa & Anthocyanins & UV & [41] \\
\hline Senegal tea & $\begin{array}{c}\text { Gymnocoronis } \\
\text { spilanthoides } \\
\text { Asteraceae }\end{array}$ & Mexico & Pyrrolizidine alkaloid esters & HPLC, MS-MS & [42] \\
\hline Tree of heaven & $\begin{array}{l}\text { Ailanthus altissimia } \\
\text { Simaroubaceae }\end{array}$ & China & Phenlypropanoids & NMR, HRESIMS & [43] \\
\hline Tuber ladder fern & $\begin{array}{c}\text { Nephrolepis cordifolia } \\
\text { Davalliaceae }\end{array}$ & Australia & 2,4-hexadien-1-ol, nonanal, thymol & GC-MS & [44] \\
\hline White bryony & $\begin{array}{l}\text { Bryonia cretica } \\
\text { Cucurbitaceae }\end{array}$ & Eurasia & Cucurbitane type triterpene & Bioassay guided fractionation, NMR & [45] \\
\hline Bracken & $\begin{array}{l}\text { Pteridium esculentum } \\
\text { Dennstaedtiaceae }\end{array}$ & Australia & Ptesculentoside, caudatoside, ptaquiloside & LC-MS & [46] \\
\hline Catsear & $\begin{array}{c}\text { Hypochaeris radicata } \\
\text { Asteraceae }\end{array}$ & Eurasia & Lignans, sesquiterpene lactones & NMR, HRMS & {$[47]$} \\
\hline $\begin{array}{l}\text { Creeping } \\
\text { buttercup }\end{array}$ & $\begin{array}{l}\text { Ranunculus repens } \\
\text { Ranunculaceae }\end{array}$ & Europe, Asia & Methyl 3,4,5-trihydroxybenzoate & 1D, 2D NMR & [48] \\
\hline Hairy buttercup & $\begin{array}{l}\text { Ranunculus sardous } \\
\text { Ranunculaceae }\end{array}$ & Europe, Asia & Ranunculin & TLC, HPTLC & [49] \\
\hline
\end{tabular}

Table 3: A summary of pharmacological activities exhibited by weeds.

\begin{tabular}{|c|c|c|c|c|c|}
\hline Common name & Botanical name & Native of & $\begin{array}{l}\text { Reported pharmacological } \\
\text { activity }\end{array}$ & Outcome of study & Reference \\
\hline Aristea & $\begin{array}{l}\text { Aristea ecklonii } \\
\quad \text { Iridaceae }\end{array}$ & West and South Africa & Antimicrobial & $\begin{array}{l}\text { Plumbagin isolated from plant exhibited } \\
\text { antimicrobial activities with MIC } 2 \mu \mathrm{g} / \mathrm{ml} \text { and } 16 \mu \mathrm{g} / \\
\mathrm{ml}\end{array}$ & {$[50]$} \\
\hline Arrow bamboo & $\begin{array}{l}\text { Pseudosasa japonica } \\
\text { Poaceae }\end{array}$ & Japan, South Korea & Antioxidant & $\begin{array}{c}\text { Leaves extract has potential to ameliorate oxidative } \\
\text { stress by improving antioxidant activity }\end{array}$ & {$[51]$} \\
\hline Bear's breeches & $\begin{array}{l}\text { Acanthus mollis } \\
\text { Acanthaceae }\end{array}$ & South West Europe & Antioxidant, anti-inflammatory & Ethanol extract inhibited NO production & {$[52]$} \\
\hline Blue spur flower & $\begin{array}{c}\text { Plectranthus ecklonii } \\
\text { Lamiaceae }\end{array}$ & South Africa & Against pancreatic cancer & $\begin{array}{l}\text { Antiproliferative effect was found to be effective } \\
\text { against BxPC3, PANC-1, Ins1-E, MICF-7, HaCat, } \\
\text { Caco-2 cell lines }\end{array}$ & {$[53]$} \\
\hline Buddleia & $\begin{array}{l}\text { Buddleja davidii } \\
\text { Buddlejaceae }\end{array}$ & China & AChE inhibitory activity & Linarin isolated from plant inhibit AChE activity & {$[54]$} \\
\hline Cape honeysuckle & $\begin{array}{l}\text { Tecomaria capensis } \\
\text { Bignoniaceae }\end{array}$ & South Africa & $\begin{array}{l}\text { Analgesic, antipyretic, anti- } \\
\text { inflammatory activities }\end{array}$ & $\begin{array}{l}\text { Methanolic extract of leaves significantly prevented } \\
\text { increase in volume of paw edema }\end{array}$ & {$[55]$} \\
\hline Cat's claw creeper & $\begin{array}{c}\text { Macfadyena unguis-cati } \\
\text { Bignoniaceae }\end{array}$ & $\begin{array}{l}\text { Central and South } \\
\text { America }\end{array}$ & Anti-inflammatory, cytotoxic & $\begin{array}{l}\text { Crude ethanol extract exhibited marked anti- } \\
\text { inflammatory and cytotoxicity against lung cancer } \\
\text { cell line }\end{array}$ & {$[56]$} \\
\hline Chocolate vine & $\begin{array}{l}\text { Akebia quinata } \\
\text { Lardizabalaceae }\end{array}$ & China, Korea, Japan & Anti-fatique agent & $\begin{array}{c}\text { Akebia extract showed marked improvement in } \\
\text { lethargic behavioral test }\end{array}$ & {$[57]$} \\
\hline Clematis & $\begin{array}{l}\text { Clematis flammula } \\
\text { Ranunculaceae }\end{array}$ & $\begin{array}{l}\text { Southern Europe and } \\
\text { Northern Africa }\end{array}$ & Cytotoxic & $\begin{array}{l}\text { Weed extract cause kinases and transcription factor } \\
\text { induction }\end{array}$ & [58] \\
\hline $\begin{array}{l}\text { Climbing spindle } \\
\text { berry }\end{array}$ & $\begin{array}{c}\text { Celastrus orbiculatus } \\
\text { Celastraceae }\end{array}$ & $\begin{array}{c}\text { Eastern Asia, Korea, } \\
\text { Japan, China }\end{array}$ & Against gastric cancer & $\begin{array}{l}\text { Compound 28-hydroxy-3-oxoolean-12-en-29-oic } \\
\text { acid inhibited the migration and invasion of gastric } \\
\text { cancer cells }\end{array}$ & [59] \\
\hline Darwin's barberry & $\begin{array}{l}\text { Berberis darwinii } \\
\text { Berberidaceae }\end{array}$ & Chile, Argentina & Alzheimer's disease & $\begin{array}{l}\text { Methanolic extract of stem bark exhibited } \\
\text { acetylcholinestrase inhibitory activity }\end{array}$ & {$[60]$} \\
\hline Elder & $\begin{array}{l}\text { Sambucus nigra } \\
\text { Caprifoliaceae }\end{array}$ & $\begin{array}{l}\text { Europe, West Asia, } \\
\quad \text { North Africa }\end{array}$ & Antioxidant & Free radical scavenging potential & [61] \\
\hline Elephant ear & $\begin{array}{l}\text { Alocasia brisbanensis } \\
\text { Araceae }\end{array}$ & Ceylon, Tahiti & Antimicrobial & $\begin{array}{c}\text { Extract showed promising antimicrobial activities } \\
\text { against Staphylococcus aureus }\end{array}$ & [62] \\
\hline False acacia & $\begin{array}{l}\text { Robinia pseudoacacia } \\
\text { Fabaceae }\end{array}$ & South Eastern USA & Antitumor & Inhibition of IL- $1 \beta$ signaling & [63] \\
\hline False tamarisk & $\begin{array}{l}\text { Myricaria germanica } \\
\text { Tamaricaceae }\end{array}$ & Eurasia & Cytotoxic & $\begin{array}{l}\text { Compound tamgermanitin exhibited potent anti } \\
\text { cancer effect }\end{array}$ & {$[64]$} \\
\hline Field horsetail & $\begin{array}{l}\text { Equisetum arvense } \\
\text { Equisetaceae }\end{array}$ & $\begin{array}{l}\text { Temperate Northern } \\
\text { Hemisphere }\end{array}$ & Antioxidant & Potent antioxidant in DPPH assay & [65] \\
\hline $\begin{array}{l}\text { Green daphne } \\
\text { laurel }\end{array}$ & $\begin{array}{l}\text { Daphne laureola } \\
\text { Thymelaeaceae }\end{array}$ & $\begin{array}{l}\text { North Africa, South } \\
\text { West Europe }\end{array}$ & Anticancer & Cytotoxic against lung cancer & {$[66]$} \\
\hline Green goddess & $\begin{array}{l}\text { Zantedeschia aethiopica } \\
\text { Araceae }\end{array}$ & South Africa & Antimicrobial & Peptides in weed exhibited antimicrobial activities & {$[67]$} \\
\hline Gypsywort & $\begin{array}{l}\text { Lycopus europaeus } \\
\text { Lamiaceae }\end{array}$ & Europe, Asia & Antimicrobial & $\begin{array}{l}\text { Compound euroabienol showed broad spectrum } \\
\text { activity }\end{array}$ & [68] \\
\hline
\end{tabular}




\begin{tabular}{|c|c|c|c|c|c|}
\hline Hop & $\begin{array}{l}\text { Humulus lupulus } \\
\text { Cannabaceae }\end{array}$ & $\begin{array}{l}\text { Europe, Western Asia, } \\
\text { North America }\end{array}$ & Osteogenic & Activity evaluated via МС3Т3-E1 cells lines & [69] \\
\hline Horned poppy & $\begin{array}{l}\text { Glaucium flavum } \\
\text { Papaveraceae }\end{array}$ & $\begin{array}{l}\text { Western Europe, } \\
\text { South Western Asia }\end{array}$ & Against breast cancer & $\begin{array}{l}\text { Bocconoline compound isolated from the plant } \\
\text { inhibit viability of cancer cells }\end{array}$ & [70] \\
\hline Houttuynia & $\begin{array}{l}\text { Houttuynia cordata } \\
\text { Saururaceae }\end{array}$ & Asia & Antiviral & $\begin{array}{c}\text { Houttuynoid B isolated from the weed prevents cell } \\
\text { entry of Zika virus }\end{array}$ & [71] \\
\hline Ivy & $\begin{array}{l}\text { Hedera helix } \\
\text { Araliaceae }\end{array}$ & Europe, North Africa & Anti-inflammatory & Inhibition of Staphylococcus aureus strain & [72] \\
\hline Jerusalem cherry & $\begin{array}{c}\text { Solanum pseudocapsicum } \\
\text { Solanaceae }\end{array}$ & South America & Acetylcholinestrase inhibitor & $\begin{array}{l}\text { Alkaloids reported in the plant exhibited AChE } \\
\text { inhibition }\end{array}$ & [73] \\
\hline Lantana & $\begin{array}{l}\text { Lantana camara } \\
\text { Verbenaceae }\end{array}$ & Tropical America & Sedative & $\begin{array}{l}\text { Essential oil from weed possess CNS depressant } \\
\text { effects }\end{array}$ & [74] \\
\hline Mexican water lily & $\begin{array}{l}\text { Nymphaea mexicana } \\
\text { Nymphaeaceae }\end{array}$ & Mexico & Anti-inflammatory & Cox-2 inhibition & [75] \\
\hline Nasturtium & $\begin{array}{l}\text { Tropaeolum majus } \\
\text { Tropaeolaceae }\end{array}$ & $\begin{array}{l}\text { Europe, America, } \\
\text { Africa, Asia }\end{array}$ & Antimicrobial & $\begin{array}{c}\text { Compound 3-[3-pyridinyl)-1,2,4-oxadiazol-5-yl] } \\
\text { benzonitrile exhibited potent antimicrobial activities }\end{array}$ & [76] \\
\hline Needlebush & $\begin{array}{l}\text { Hakea sericea } \\
\text { Proteaceae }\end{array}$ & Australia & Cytotoxic & Extract inhibited MCF-7 cell line & [77] \\
\hline Old man's beard & $\begin{array}{l}\text { Clematis vitalba } \\
\text { Ranunculaceae }\end{array}$ & $\begin{array}{l}\text { Europe, South West } \\
\text { Asia }\end{array}$ & Antinociceptive and antipyretic & Vitalboside isolated from weed exerted action & {$[78]$} \\
\hline Pig's ear & $\begin{array}{l}\text { Cotyledon orbiculata } \\
\text { Crassulaceae }\end{array}$ & Africa & Anticonvulsant & $\begin{array}{c}\text { Aqueous and methanolic extracts showed } \\
\text { prominent effects on gabaergic and glutaminergic } \\
\text { mechanisms }\end{array}$ & [79] \\
\hline Pink ragwort & $\begin{array}{l}\text { Senecio latifolius } \\
\text { Asteraceae }\end{array}$ & South Africa & Cytotoxic & $\begin{array}{l}\text { Cytotoxicity in HepG2 cells caused depletion of } \\
\text { cellular GSH }\end{array}$ & {$[80]$} \\
\hline Rough horsetail & $\begin{array}{l}\text { Equisetum hyemale } \\
\text { Equisetaceae }\end{array}$ & $\begin{array}{l}\text { Temperate Northern } \\
\text { Hemisphere }\end{array}$ & Antitrypanosomal & n-butanol fraction exert antiprotozoal effect & [81] \\
\hline Royal fern & $\begin{array}{l}\text { Osmunda regalis } \\
\text { Osmundaceae }\end{array}$ & Europe, India, Africa & $\begin{array}{l}\text { Inhibition of head and neck } \\
\text { cancer cell proliferation }\end{array}$ & $\begin{array}{c}\text { Extract revealed growth inhibiting effect on } \mathrm{HLaC78} \\
\text { and } \mathrm{FaDu}\end{array}$ & [82] \\
\hline Tree privet & $\begin{array}{l}\text { Ligustrum lucidum } \\
\text { Oleaceae }\end{array}$ & China & Hepatocellular carcinoma & Inactivation of PL3K/Akt pathway & [83] \\
\hline Tutsan & $\begin{array}{l}\text { Hypericum androsaemum } \\
\text { Clusiaceae }\end{array}$ & $\begin{array}{l}\text { South and Western } \\
\text { Europe }\end{array}$ & Anti-lipid peroxidation & n-hexane fraction exerted desired activity & {$[84]$} \\
\hline Chingma lantern & $\begin{array}{l}\text { Abutilon theophrasti } \\
\text { Malvaceae }\end{array}$ & Temperate region & Antibacterial & $\begin{array}{l}\text { Extract showed activity against Staphylococcus } \\
\text { aureus }\end{array}$ & [85] \\
\hline Black night shade & $\begin{array}{l}\text { Solanum nigrum } \\
\text { Solanaceae }\end{array}$ & North Western Africa & Antitumor & Active against breast cancer cell line MCF7 & {$[86]$} \\
\hline Broad leaved dock & $\begin{array}{l}\text { Rumex obtusifolius } \\
\text { Polygonaceae }\end{array}$ & Eurasia & Hypoglycemic & $\begin{array}{l}\text { Ethanolic extract improved glucose tolerance in } \\
\text { rabbits }\end{array}$ & [87] \\
\hline $\begin{array}{l}\text { Broad leaved } \\
\text { fleabane }\end{array}$ & $\begin{array}{l}\text { Conyza sumatrensis } \\
\text { Asteraceae }\end{array}$ & South America & Antiplasmodial & Study confirmed the traditional use of weed & [88] \\
\hline $\begin{array}{l}\text { Broad leaved } \\
\text { plantain }\end{array}$ & $\begin{array}{l}\text { Plantago major } \\
\text { Plantaginaceae }\end{array}$ & Eurasia & Potential wound healer & $\begin{array}{l}\text { Showed activity against hyaluronidase and } \\
\text { collangenase enzymes }\end{array}$ & [89] \\
\hline Chick weed & $\begin{array}{l}\text { Stellaria media } \\
\text { Caryophyllaceae }\end{array}$ & India & Antifungal & $\begin{array}{l}\text { Peptides in weed were responsible for its potent } \\
\text { activity }\end{array}$ & [90] \\
\hline Cleavers & $\begin{array}{l}\text { Galium aparine } \\
\text { Rubiaceae }\end{array}$ & Temperate zone & Immunomodulator & $\begin{array}{l}\text { Ethanolic extract stimulated immunocompetent } \\
\text { blood cells }\end{array}$ & [91] \\
\hline Dandelion & $\begin{array}{l}\text { Taraxacum officinale } \\
\text { Asteraceae }\end{array}$ & Africa & Antioxidant & $\begin{array}{l}\text { Extract from leaf provide protection against free } \\
\text { radical mediated oxidative stress }\end{array}$ & [92] \\
\hline Father & $\begin{array}{l}\text { Chenopodium album } \\
\text { Amaranthaceae }\end{array}$ & Temperate zone & Antioxidant & $\begin{array}{l}\text { Weed showed protection against mercury induced } \\
\text { oxidative stress }\end{array}$ & [93] \\
\hline Galinsoga & $\begin{array}{l}\text { Galinsoga parviflora } \\
\text { Asteraceae }\end{array}$ & Tropical America & Photocarcinogenesis & $\begin{array}{c}\text { Caffeic acid derivative protect dermal UVA-induced } \\
\text { oxidative stress }\end{array}$ & [94] \\
\hline Hedge mustard & $\begin{array}{l}\text { Sisymbrium officinale } \\
\text { Brassicaceae }\end{array}$ & Southern Europe & $\begin{array}{l}\text { Inhibition of oxidative } \\
\text { mutagenicity }\end{array}$ & Polyphenols in weed exhibited desired action & [95] \\
\hline Hemlock & $\begin{array}{l}\text { Conium maculatum } \\
\text { Apiaceae }\end{array}$ & Temperate region & Antimicrobial & $\begin{array}{l}\text { Essential oils reported in weed showed activity } \\
\text { against } P \text { seudomonas aeruginosa }\end{array}$ & [96] \\
\hline Manuka & $\begin{array}{l}\text { Leptospermum scoparium } \\
\text { Myrtaceae }\end{array}$ & $\begin{array}{l}\text { New Zealand, South } \\
\text { East Australia }\end{array}$ & Antibacterial & $\begin{array}{l}\text { Oils exhibited activity against gram negative } \\
\text { pathogens }\end{array}$ & [97] \\
\hline Nettle & $\begin{array}{l}\text { Urtica urens } \\
\text { Urticaceae }\end{array}$ & Europe & Anxiolytic & $\begin{array}{l}\text { Extract increased the time spent in bright-lit } \\
\text { chamber of light/dark box pharmacological model }\end{array}$ & [98] \\
\hline Pennyroyal & $\begin{array}{l}\text { Mentha pulegium } \\
\text { Lamiaceae }\end{array}$ & Northern Africa & Antidiabetic & $\begin{array}{c}\text { Aqueous extract revealed improvement of glucose } \\
\text { tolerance in in-vivo rat model }\end{array}$ & [99] \\
\hline Red dead nettle & $\begin{array}{l}\text { Lamium purpureum } \\
\text { Lamiaceae }\end{array}$ & Eurasia & Haemostatic activity & $\begin{array}{l}\text { Extracts showed promising results in haemostatic } \\
\text { test }\end{array}$ & [100] \\
\hline Scarlet pimpernel & $\begin{array}{l}\text { Anagallis arvensis } \\
\text { Primulaceae }\end{array}$ & Northern Africa & Molluscicidal & $\begin{array}{c}\text { Aqueous leaf extract showed activity against } \\
\text { Schistosoma mansoni }\end{array}$ & [101] \\
\hline Scotch thistle & $\begin{array}{l}\text { Cirsium vulgare } \\
\text { Asteraceae }\end{array}$ & Europe & Hepatoprotective & $\begin{array}{l}\text { Hexane extract showed anti-necrotic and anti- } \\
\text { cholestatic effects }\end{array}$ & [102] \\
\hline $\begin{array}{l}\text { Scrambling } \\
\text { speedwell }\end{array}$ & $\begin{array}{l}\text { Veronica persica } \\
\text { Plantaginaceae }\end{array}$ & Eurasia, America & Antiviral & $\begin{array}{l}\text { Extract showed synergistic activity in combination } \\
\text { with acyclovir anti-HSV therapy }\end{array}$ & [103] \\
\hline
\end{tabular}




\begin{tabular}{|c|c|c|c|c|c|}
\hline Selfheal & $\begin{array}{l}\text { Prunella vulgaris } \\
\text { Lamiaceae }\end{array}$ & Eurasia, America & Inhibition of IHNV infection & Ursolic acid decrease cytopathic effect and viral titer & [104] \\
\hline Sow thistle & $\begin{array}{l}\text { Sonchus oleraceus } \\
\text { Asteraceae }\end{array}$ & Asia & Nephroprotective & $\begin{array}{l}\text { Extract showed desired effect by inhibiting ischemia } \\
\text { reperfusion in rats }\end{array}$ & [105] \\
\hline Water pepper & $\begin{array}{l}\text { Persicaria hydropiper } \\
\text { Polygonaceae }\end{array}$ & Eurasia & Anti-inflammatory & Extract showed desired therapeutic effect & [106] \\
\hline Yarrow & $\begin{array}{l}\text { Achillea millefolium } \\
\text { Asteraceae }\end{array}$ & Eurasia, North America & Antibabesial activity & Different extract were active against Brucella canis & [107] \\
\hline Woolly mullein & $\begin{array}{l}\text { Verbascum thapsus } \\
\text { Scrophulariaceae }\end{array}$ & Eurasia & Antimicrobial & $\begin{array}{l}\text { Ethanolic extract were potent against gram positive } \\
\text { bacteria }\end{array}$ & [108] \\
\hline Wild teasel & $\begin{array}{l}\text { Dipsacus fullonum } \\
\text { Caprifoliaceae }\end{array}$ & Australia & Antibacterial & $\begin{array}{l}\text { Compounds isolated from root exhibited activity } \\
\text { against Staphylococcus aureus }\end{array}$ & [109] \\
\hline Cocklebur & $\begin{array}{l}\text { Xanthium strumarium } \\
\text { Asteraceae }\end{array}$ & Temperate zone & Hepatocellular carcinoma & $\begin{array}{c}\text { Weed induce apoptosis in } \mathrm{HCC} \text { cell lines in a dose } \\
\text { dependent manner }\end{array}$ & [110] \\
\hline
\end{tabular}

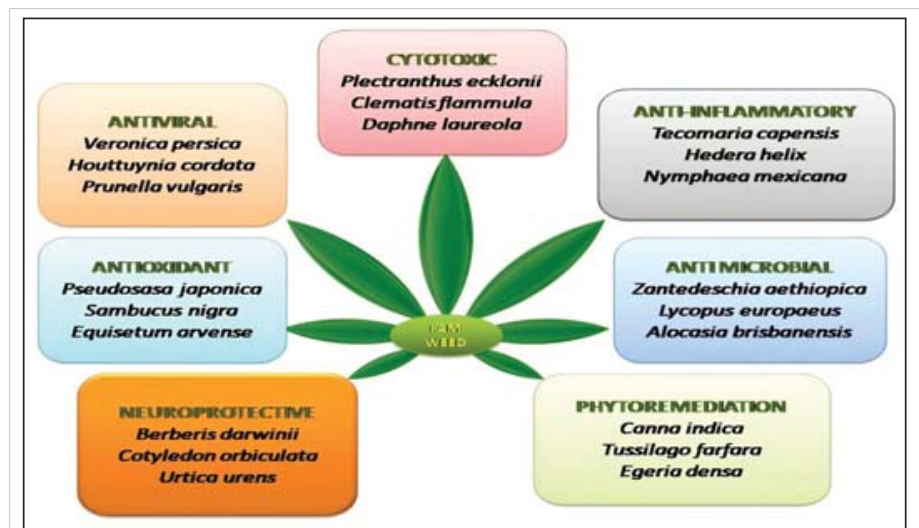

Figure 1: Therapeutic potential of weeds.

include Clematis flammula [58], Hakea sericea (Needlebush) [77], Robinia pseudoacacia (False acacia) [63], Daphne laureola [66]. Myricaria germanica (False tamarisk) [64], Senecio latifolius (Pink ragwort) [80]. Osmunda regalis (Royal fern) [82]. Parvifloron D isolated from Plectranthus ecklonii via flash dry column chromatography exhibited antiproliferative effects against pancreatic cancer when evaluated against HaCat, BxPC3, Caco-2, MCF-7, Ins1-E and PANC-1 cell lines [53]. Aqueous extract of weed Solanum nigrum at concentration of $10 \mathrm{~g} / \mathrm{l}$ caused $43 \%$ cytotoxicity in MCF7 cell line by inhibiting migration, suppression of hexokinase and pyruvate kinase [86]. Triterpene (28-Hydroxy-3-oxoolean-12-en-29-oic acid) present in Celastrus orbiculatus showed inhibitory activity on SGC-7901 and BGC-823 cells lines [59]. Bocconoline alkaloid isolated from dried roots of Glaucium flavum (Horned poppy) exhibited cytotoxicity with $\mathrm{IC}_{50}$ value of $7.8 \mu \mathrm{M}$ [70].

Antimicrobial weeds: Invasive weed Aristea ecklonii containing Plumbagin exhibited antimicrobial activity with minimum inhibitory concentration between $2 \mu \mathrm{g} / \mathrm{ml}$ and 16 $\mu \mathrm{g} / \mathrm{ml}[50]$. Antimicrobial peptides isolated from arum lily (Zantedeschia aethiopica) exhibited potent antimicrobial activity [67]. Euroabienol (abietane-type diterpenoid) isolated from fruits of Lycopus europaeus exhibited broad spectrum antimicrobial activity [68]. Compounds 3-[3-(3-pyridinyl)-1,2,4-oxadiazol-5-yl] benzonitrile and [3,5-Bis (1,1-dimethylethyl)-4-hydroxyphenyl] isolated from weed Tropaeolum tuberosum when tested against Candida tropicalis exhibited antifungal activities with MICs of $100 \mu \mathrm{M}$ and $50 \mu \mathrm{M}$ [76]. Extracts obtained from leaves of weed Abutilon theophrasti elicit antimicrobial potential against Staphylococcus aureus, Salmonella, Streptococcus and E. coli species [85]. Essential oils isolated from weeds Conium maculatum, Leptospermum scoparium showed antimicrobial activity against several strains of Pseudomonas aeruginosa $[96,97]$. Ethanolic extracts of woolly mullein reported positive against gram positive bacteria (Bacillus cereus) [108]. Phenolic compounds from Dipsacus fullonum exerted inhibitory effects on Staphylococcus aureus DSM 799 and E. coli ATCC 10536 strains [109].

Antioxidant weeds: Strong antioxidant activity was reported by ferulic acid derived from leaves of weed Pseudosasa japonica when evaluated using DPPH (54 \%) and ABTS (65\%) [51]. Antioxidant potential of Taraxacum officinale was determined using in vitro methods (DPPH, ABTS, FRAP). The ABTS method reveled that antioxidant activity was $156 \pm 5.28 \mu \mathrm{g} / \mathrm{ml}$ [92]. Other potential antioxidant weed includes Acanthus mollis [52], Sambucus nigra [61], Equisetum arvense [65].

Anti-inflammatory weeds: A study by Akhtar, et al. 2019 investigated the anti-inflammatory properties of Hedera helix and its major compounds on Staphylococcus aureus induced inflammation in mice. Hederacoside-C isolated from weed exerted profound anti-inflammatory effects [72]. Mexican water lily (Nymphaea mexicana) was found to be potent COX-2 inhibitor [75]. Active compounds isolated from aerial parts of weed Clematis vitalba when evaluated in vivo against carrageenan, serotonin, PGE- 2 induced hind paw edema showed antinociceptive and antipyretic effects [78]. Methanolic extract of leaves of Tecomaria capensis significantly prevented increase in volume of paw edema [55]. Extract of Persicaria hydropiper exerted marked anti-inflammatory effects [106]. Aqueous extract alongwith compounds (calceorioside B, homoplantaginin, plantamajoside) isolated from the aerial parts of Plantago major showed inhibition against hyaluronidase enzyme [89].

Antiviral weeds: Methanolic extract of scrambling speedwell weed (Veronica persica) reported potent activity against herpes simplex viruses and synergistic activity in combination with acyclovir anti-HSV therapy [103]. Ursolic 
acid isolated form weed Prunella vulgaris inhibited IHNV infection in aquaculture with an inhibitory concentration of $99.3 \%$ at $100 \mathrm{mg} / \mathrm{l}[104]$.

Weeds acting on CNS: Methanolic extract of stem bark of darwin's barberry (Berberis darwinii) inhibited acetylcholinestrase in vitro with IC $_{50}$ value of $1.23 \pm 0.05$ microg/ $\mathrm{mL}$ thereby provide relief in alzheimer's disease [60]. Alkaloid solanocapsine isolated from weed Solanum pseudocapsicum reported to inhibit activity of enzyme acetylcholinestrase [73]. Nettle (Urtica urens) exhibited anxiolytic activity in mice when evaluated using hole board test, light-dark box test and rota rod test. Extract showed increased head-dip duration and head-dip counts in hole board test [98]. Aqueous (50-400 mg/ kg i.p.) and methanolic extracts (100-400 mg/kg i.p.) of Pig's ear (Cotyledon orbiculata) exhibited anticonvulsant activity which predominantly delayed onset of seizures induced by $\mathrm{N}$-methyl-dl-aspartic, bicuculline, picrotoxin in mice models [79].

Other pharmacological activities of weeds: Aqueous extract of Akebia quinata showed positive effect against fatigue in mice exposed to chronic restraint stress when evaluated using forced swimming behavioral test, sucrose preference and open field tests [57]. n-butanol fraction of weed Equisetum hyemale exerted antiprotozoal effects against Trypanosoma evansi trypomastigotes after nine hours exposure [81]. Chen, et al. 2019 reported osteogenic activities of Humulus lupulus in MC3T3-E1 cell lines [69]. Ethanolic extract of weed Galium aparine stimulated the transformational activity of immunocompetent blood cells in vitro [91]. Aqueous extract of aerial parts of Mentha pulegium $(20 \mathrm{mg} / \mathrm{kg}$ ) showed antihyperglycemic effect by marked improvement in oral glucose tolerance test in streptozotocin induced diabetic rats [99]. Butanolic extracts of aerial parts of Lamium album and Lamium pupureum showed haemostatic activity in wistar rats when evaluated by tail bleeding time determination and acenocoumarol carrageenan test compared to vitamin $\mathrm{K}$ [100]. Anagallis arvensis (Scarlet pimpernel) leaf extract showed molluscicidal activity against Biomphalaria alexandrina at $\mathrm{LC}_{50} 37.9 \mathrm{mg} / \mathrm{l}$ and $\mathrm{LC}_{90} 48.3 \mathrm{mg} / \mathrm{l}$ [101]. Hexane extract rich in lupeol acetate of weed scotch thistle (Cirsium vulgare) prevented carbon tetrachloride induced liver damage in rats by diminishing lipid peroxidation and nitric oxide levels [102]. Extracts of Sonchus oleraceus (Sow thistle) were reported to be nephroprotective against kidney ischemia reperfusion injury in wistar rats [105]. Water extract, ethanol extract, hexane/acetone extract obtained from Achillea millefolium (Yarrow) were effective against Babesia canis parasite at 2 $\mathrm{mg} / \mathrm{ml}$ concentration [107].

\section{Other potential applications of weeds established in New Zealand}

A large number of weed communities has been reported to clean environment through phytoremediation process and act as bioindicators (Figure 2). Phytoremediation is

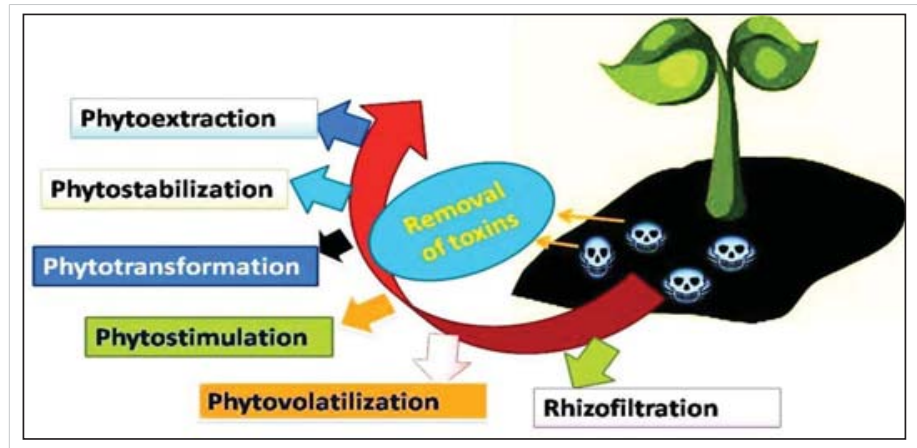

Figure 2: An overview of Phytoremediation process.

described as a process of eradicating toxic contaminants from soil, water and air. This process involves phytoextraction (harvesting of biomass), phytostabilization (contaminants stabilized into less toxic compounds), phytotransformation (chemical modification of contaminants), phytostimulation (rhizosphere degradation), phytovolatilization (conversion of toxic compounds into volatile form) and rhizofiltration (filtration through roots) [111]. Arum lily (Zantedeschia aethiopica) acts as micropollutant removal by removing accumulation of copper, zinc, carbamazepine and linear alkylbenxene sulphonates [112]. Bladderwort (Utricularia gibba) [113], canna lilly (Canna indica) [114], coltsfoot (Tussilago farfara) [115], egeria (Egeria densa) [116], giant buttercup (Ranunculus acris) [117], broom (Cytisus scoparius) [118], annual poa (Poa annua) have been involved in removing toxic metals (chromium, cadmium, zinc, lead) from the environment [119]. Parrot feather (Myriophyllum aquaticum) aids in removing antibiotic (tetracycline) from water [120]. Oxeye daisy (Leucanthemum vulgare) potentiated crude oil phytoremediation and used in eliminating pollution from environment [121]. Apart from these properties weeds have also been found to be employed in other industries e.g. buffalo grass weed (Stenotaphrum secundatum) used in turf grass industry [122]. Mucoadhesive properties of water soluble gum obtained from Hakea gibbosa added in sustained release dosage forms [123]. Silver nanoparticles having average particle size $20 \mathrm{~nm}$ synthesized from Cestrum nocturnum showed more antioxidant potential as compared to vitamin C alongwith strong antibacterial activity against Vibrio cholerae with MIC of $16 \mu \mathrm{g} / \mathrm{ml}$ [124]. Organic fertilizer manufactured via aquatic weed Salvinia molesta when evaluated using FT-IR, plant bioassay test for determination of its fertilizer value and chemical composition showed promising results as vermicompost [125]. Eragrostis species (E. capensis, E. curvula) and grass Stenotaphrum secundatum exhibited drought resistant ability $[126,127]$.

Besides the therapeutic potential exhibited by weeds, toxicity profile should be taken into consideration while exploring them. Equisetum arvense (Field horsetail) exerted hepatotoxicity in rats [128], weeds like Zantedeschia aethiopica (Arum lily), Conium maculatum (Hemlock), Solanum nigrum (Black night shade) are considered poisonous in New Zealand 


\begin{tabular}{|l|}
\hline Important abbreviations used \\
\hline ABTS \\
\hline COX \\
\hline DPPH \\
\hline ESI \\
\hline FAB \\
\hline FRAP \\
\hline FTIR \\
\hline GC \\
\hline GSH \\
\hline HepG2 \\
\hline HPLC \\
\hline HPTLC \\
\hline HRESIFTMS \\
\hline HR-ESI-MS \\
\hline HSV \\
\hline IC \\
\hline IHNV \\
\hline LC-ESIMS \\
\hline MCF7, SGC-7901, BGC-823 cells, BxPC3, PANC-1, Ins1-E, MICF-7, HaCat, Caco-2, \\
\hline HCC, HLaC78, FaDu \\
\hline MS \\
\hline NMR \\
\hline NO \\
\hline PGE-2 \\
\hline TLC \\
\hline UAE \\
\hline UV \\
\hline UVA \\
\hline
\end{tabular}

[129]. Hedera helix (Ivy) caused contact dermatitis [130], Lantana camara exerted in vivo cell toxicity [131], Xanthium strumarium (Cocklebur) responsible for causing poisoning in cattle [132].

\section{Conclusion}

Humans define weeds as per their appropriateness and understanding of the plant. A plant investigated as weed in some region may be a plant of medicinal importance for another region. The usefulness of weeds has been ignored by humans for long time because of their invasive growth, competitors of genuine crop and no economic value. This human behaviour might be developed over time due to lack of proper knowledge of phytochemical screening as well as therapeutic potential of weeds. Weeds are the sources of human food, fodder in agriculture, shelter for some animals, helpful against soil erosion, indicators of soil nutrients, as well as sources of commercially important essential oils. In this era weeds have been extensively explored for their immense phytopharmacological prospects. It is evidenced that weeds have been sources of potential targets for different pathological conditions. However there is need of more scientific and clinical investigations required in assessment of toxicity profile to get the maximum potential of weeds. Weeds have protective role in environment as a component of phytoremediation and for sustainable ecosystem. Because of immense therapeutic potential implicit by weeds a new chain of thoughts emerge in our mind to consider the value 2,2'-azino-bis(3-ethylbenzothiazoline-6-sulfonic acid)

Cyclooxygenase

2,2-diphenyl-1-picrylhydrazyl

Electrospray ionization

Fast atom bombardment

Fluorescence recovery after photobleaching

Fourier-transform infrared spectroscopy

Gas chromatography

Glutathione

Hepatoblastoma cells

High-performance liquid chromatography

High-performance thin-layer chromatography

High resolution electrospray ionization fourier transform mass spectrometry

High-resolution electrospray ionization mass spectrometry

Herpes simplex virus

Inhibitory concentration

Infectious hematopoietic necrosis virus

Liquid chromatography electrospray ionisation mass spectroscopy

Cancer cell lines

Mass spectroscopy

Nuclear magnetic resonance

Nitric oxide

Prostaglandin E2

Thin layer chromatography

Ultrasound-assisted extraction

Ultraviolet spectroscopy

Ultra violet radiation

of these important plants so called 'weeds'. Are they need to be redefined or we need to rethink the concept of weeds? It is clear from the studies documented in this review that the approach of whether a plant is wanted or not should depends on its pharmacological potential and role in ecosystem other than merely the competitive effect of plant with the particular crop. Further advancements are required in order to spin the concept of weeds into therapeutic weeds.

\section{References}

1. Jethro Tull. Horse hoeing husbandary, Berkshire. MDCC, 33; 1731.

2. Crawley MJ. Biodiversity. In: Crawley, M.J., (ed.) Plant Ecology, 2nd Edn. Blackwell Scientific, Oxford; 1997.

3. Aldrich RJ, Kremer RJ. Principles in weed management. lowa State University Press; 1997.

4. Rao VS. Principles of Weed Science, 2nd Edn, Science Publishers, Enfield, New Hampshire, USA; 2000.

5. Oudhia P. Medicinal weeds in rice fields of Chhattisgarh, India International Rice Research Notes. 1999; 24:40.

6. Gibbons DW, Bohan DA, Rothery P, Stuart RC, Haughton AJ, et al. Weed seed resources for birds in fields with contrasting conventional and genetically modified herbicide-tolerant crops. Proceedings of the Royal Society B: Biological Sciences. 2006; 273: 1921-1928. PubMed: https://www.ncbi.nlm.nih.gov/pubmed/16822753

7. Hawes C, Haughton AJ, Bohan DA, Squire GR. Functional approaches for assessing plant and invertebrate abundance patterns in arable systems. Basic and Applied Ecology. 2009; 10: 34-42.

8. Kromp B. Carabid beetles in sustainable agriculture: a review on pest 
control efficacy, cultivation impacts and enhancement. Agriculture, Ecosystems \& Environment. 1999; 74: 187-228.

9. Evans CL. The war on weeds in the Prairie west: an environmental history. University of Calgary Press; 2002.

10. Popay I, Champion P, James T. An Illustrated Guide to Common Weeds of New Zealand. 3rd Edn. New Zealand Plant Protection Society. 2010.

11. Hsieh YS, Harris PJ. Structures of xyloglucans in primary cell walls of gymnosperms, monilophytes (ferns sensu lato) and lycophytes. Phytochemistry. 2012; 79: 87-101.

12. Rasanen RM, Hieta JP, Immanen J, Nieminen K, Haavikko R, et al Chemical profiles of birch and alder bark by ambient mass spectrometry. Anal Bioanal Chem. 2019; 411: 7573-7583.

13. Collett MG, Taylor SM. Photosensitising toxins in alligator weed (Alternanthera philoxeroides) likely to be anthraquinones. Toxicon. 2019; 167: 172-173.

PubMed: https://www.ncbi.nlm.nih.gov/pubmed/31226258

14. Alipieva K, Evstatieva L, Handjieva N, Popova S. Comparative analysis of the composition of flower volatiles from Lamium $L$. species and Lamiastrum galeobdolon Heist. ex Fabr. Zeitschrift fur Naturforschung C. $2003 ; 58: 779-782$

15. Masi M, Di Lecce R, Tuzi A, Linaldeddu BT, Montecchio L, et al. Hyfraxinic Acid, a Phytotoxic Tetrasubstituted Octanoic Acid, Produced by the Ash (Fraxinus excelsior L.) Pathogen Hymenoscyphus fraxineus Together with Viridiol and Some of Its Analogues. J Agric Food Chem. 2019; 67: 13617-13623.

PubMed: https://www.ncbi.nlm.nih.gov/pubmed/31661270

16. Metlicar V, Vovk I, Albreht A. Japanese and Bohemian Knotweeds as Sustainable Sources of Carotenoids. Plants. 2019; 8: 384.

PubMed: https://www.ncbi.nlm.nih.gov/pmc/articles/PMC6843863/

17. Alamzeb M, Omer M, Ur-Rashid M, Raza M, Ali S, et al. NMR, Novel Pharmacological and In Silico Docking Studies of Oxyacanthine and Tetrandrine: Bisbenzylisoquinoline Alkaloids Isolated from Berberis glaucocarpa Roots. J Anal Methods Chem. 2018; 7692913. PubMed: https://www.ncbi.nlm.nih.gov/pubmed/29888027

18. Kitryte V, Narkeviciute A, Tamkute L, Syrpas M, Pukalskiene M, et al. Consecutive high-pressure and enzyme assisted fractionation of blackberry (Rubus fruticosus L.) pomace into functional ingredients: Process optimization and product characterization. Food Chem. 2020 312: 126072.

PubMed: https://www.ncbi.nlm.nih.gov/pubmed/31893552

19. Weretilnyk EA, Bednarek S, McCue KF, Rhodes D, Hanson AD. Comparative biochemical and immunological studies of the glycine betaine synthesis pathway in diverse families of dicotyledons. Planta. 1989; 178: 342-352.

20. Mithofer A, Reichelt M, Nakamura Y. Wound and insect-induced jasmonate accumulation in carnivorous Drosera capensis: two sides of the same coin. Plant Biol. 2014; 16: 982-987.

PubMed: https://www.ncbi.nlm.nih.gov/pubmed/24499476

21. Małajowicz J, Kusmirek S. Structure and properties of ricin-the toxic protein of Ricinus communis. Postepy Biochemii. 2019; 65: 03-108. PubMed: https://www.ncbi.nlm.nih.gov/pubmed/31642648

22. Cruz-Salas CN, Prieto C, Calderon-Santoyo M, Lagarón JM, RagazzoSanchez JA. Micro-and Nanostructures of Agave Fructans to Stabilize Compounds of High Biological Value via Electrohydrodynamic Processing. Nanomaterials. 2019; 9:1659.

PubMed: https://www.ncbi.nlm.nih.gov/pubmed/31766573

23. Sendker J, Ellendorff T, Holzenbein A. Occurrence of benzoic acid esters as putative catabolites of prunasin in senescent leaves of
Prunus laurocerasus. J Nat Prod. 2016; 79: 1724-1729.

PubMed: https://www.ncbi.nlm.nih.gov/pubmed/27331617

24. Bridi R, Giordano A, Penailillo MF, Montenegro G. Antioxidant Effect of Extracts from Native Chilean Plants on the Lipoperoxidation and Protein Oxidation of Bovine Muscle. Molecules. 2019; 24: 3264. PubMed: https://www.ncbi.nlm.nih.gov/pubmed/31500282

25. Ouyang MA, He ZD, Wu CL. Anti-oxidative activity of glycosides from Ligustrum sinense. Natural product research. 2003; 17: 381-387. PubMed: https://www.ncbi.nlm.nih.gov/pubmed/14577686

26. Ben Ammar R, Miyamoto T, Chekir-Ghedira L, Ghedira K, LacailleDubois MA. Isolation and identification of new anthraquinones from Rhamnus alaternus $L$ and evaluation of their free radical scavenging activity. Natural product research. 2019; 33: 280-286.

PubMed: https://pubmed.ncbi.nlm.nih.gov/29533086/

27. Matouskova M, Jurova J, Grulova D, Wajs-Bonikowska A, Renco M, Sedlak V, Poracova J, Gogalova Z, Kalemba D. Phytotoxic Effect of Invasive Heracleum mantegazzianum Essential Oil on Dicot and Monocot Species. Molecules. 2019; 24: 425.

PubMed: https://www.ncbi.nlm.nih.gov/pmc/articles/PMC6384721/

28. Lachowicz S, Oszmianski J. Profile of Bioactive Compounds in the Morphological Parts of Wild Fallopia japonica (Houtt) and Fallopia sachalinensis (F. Schmidt) and Their Antioxidative Activity. Molecules. 2019; 24: 1436.

PubMed: https://www.ncbi.nlm.nih.gov/pubmed/30979044

29. Liu QR, Li J, Zhao XF, Xu B, Xiao XH, et al. Alkaloids and phenylpropanoid from Rhizomes of Arundo donax L. Natural Product Res. 2019: 1-6.

30. Ikbal C, Habib B, Hichem BJ, Monia BH, Habib BH, et al. Purification of a natural insecticidal substance from Cestrum parqui (Solanaceae). Pak J Biol Sci. 2007; 10: 3822-3828.

PubMed: https://www.ncbi.nlm.nih.gov/pubmed/19090236

31. Mandim F, Barros L, Heleno SA, Pires TC, Dias MI, Alves MJ, Santos PF, Ferreira IC. Phenolic profile and effects of acetone fractions obtained from the inflorescences of Calluna vulgaris (L.) Hull on vaginal pathogenic and non-pathogenic bacteria. Food \& Function. 2019; 10 : 2399-2407.

PubMed: https://www.ncbi.nlm.nih.gov/pubmed/31049501

32. Zhang T, Liu H, Bai X, Liu P, Yang Y, et al. Fractionation and antioxidant activities of the water-soluble polysaccharides from Lonicera japonica Thunb. International journal of biological macromolecules. 2019. PubMed: https://www.ncbi.nlm.nih.gov/pubmed/31739015

33. Matthaus B, Ozcan MM. Fatty acid, tocopherol and squalene contents of Rosaceae seed oils. Botanical studies. 2014; 55:48.

PubMed: https://www.ncbi.nlm.nih.gov/pmc/articles/PMC5432826/

34. Jo MS, Yu JS, Lee JC, Lee S, Cho YC, et al. Lobatamunsolides A-C, Norlignans from the Roots of Pueraria lobata and their Nitric Oxide Inhibitory Activities in Macrophages. Biomolecules. 2019; 9:755. PubMed: https://www.ncbi.nlm.nih.gov/pubmed/31757072

35. Fu Y, Li F, Ding Y, Li HY, Xiang XR, et al. Polysaccharides from loquat (Eriobotrya japonica) leaves: Impacts of extraction methods on their physicochemical characteristics and biological activities. Int $\mathrm{J}$ Biol Macromolecules. 2020.

PubMed: https://www.ncbi.nlm.nih.gov/pubmed/31923490

36. Chu MJ, Du YM, Liu XM, Yan N, Wang FZ, et al. Extraction of proanthocyanidins from chinese wild rice (zizania latifolia) and analyses of structural composition and potential bioactivities of different fractions. Molecules. 2019; 24:1681.

PubMed: https://www.ncbi.nlm.nih.gov/pubmed/31052148

37. Dong LM, Zhang M, Xu QL, Zhang Q, Luo B, et al. Two new thymo 
derivatives from the roots of Ageratina adenophora. Molecules. 2017 22: 592.

PubMed: https://www.ncbi.nlm.nih.gov/pmc/articles/PMC6154539/

38. Zhang ZP, Shen CC, Gao FL, Wei H, Ren DF, et al. Isolation, purification and structural characterization of two novel water-soluble polysaccharides from Anredera cordifolia. Molecules. 2017; 22: 1276. PubMed: https://www.ncbi.nlm.nih.gov/pubmed/28769023

39. Priolo N, Del Valle SM, Arribere MC, López L, Caffini N. Isolation and characterization of a cysteine protease from the latex of Araujia hortorum fruits. Journal of Protein Chemistry. 2000; 19: 39-49.

40. Akihara Y, Kamikawa S, Harauchi Y, Ohta E, Nehira T, et al. HPLC profiles and spectroscopic data of cassane-type furanoditerpenoids. Data in brief. 2018; 21:1076-88.

PubMed: https://www.ncbi.nlm.nih.gov/pubmed/30450403

41. Jordheim M, Calcott K, Gould KS, Davies KM, Schwinn KE, et al. High concentrations of aromatic acylated anthocyanins found in cauline hairs in Plectranthus ciliatus. Phytochemistry. 2016; 128:27-34.

PubMed: https://www.ncbi.nlm.nih.gov/pubmed/27165277

42. Boppre M, Colegate SM. Recognition of pyrrolizidine alkaloid esters in the invasive aquatic plant Gymnocoronis spilanthoides (Asteraceae). Phytochemical Analysis. 2015; 26: 215-225.

43. Du YQ, Yan ZY, Chen JJ, Wang XB, Huang XX, et al. The identification of phenylpropanoids isolated from the root bark of Ailanthus altissima (Mill.) Swingle. Nat Product Res. 2019: 1-8.

PubMed: https://www.ncbi.nlm.nih.gov/pubmed/31315448

44. El-Tantawy ME, Shams MM, Afifi MS. Chemical composition and biological evaluation of the volatile constituents from the aerial parts of Nephrolepis exaltata (L.) and Nephrolepis cordifolia (L.) C. Presl grown in Egypt. Natural product research. 2016; 30: 1197-201.

PubMed: https://www.ncbi.nlm.nih.gov/pubmed/26211503

45. Matsuda H, Nakashima S, Abdel-Halim OB, Morikawa T, Yoshikawa M. Cucurbitane-type triterpenes with anti-proliferative effects on U937 cells from an egyptian natural medicine, Bryonia cretica: structures of new triterpene glycosides, bryoniaosides A and B. Chemical and Pharmaceutical Bulletin. 2010; 58: 747-51.

PubMed: https://www.ncbi.nlm.nih.gov/pubmed/20460809

46. Kisielius V, Lindqvist DN, Thygesen MB, Rodamer M, Hansen HC Rasmussen LH. Fast LC-MS quantification of ptesculentoside, caudatoside, ptaquiloside and corresponding pterosins in bracken ferns. J Chromatography B. 2020: 121966.

PubMed: https://pubmed.ncbi.nlm.nih.gov/31931331

47. Shulha O, Çiçek SS, Wangensteen H, Kroes J, Mäder M, et al Lignans and sesquiterpene lactones from Hypochaeris radicata subsp. neapolitana (Asteraceae, Cichorieae). Phytochemistry. 2019; 165: 112047.

48. Khan WN, Lodhi MA, Ali I, Azhar-Ul-Haq, Malik A, et al. New natural urease inhibitors from Ranunculus repens. J Enzyme Inhibition Med Chem. 2006; 21: 17-19.

PubMed: https://www.ncbi.nlm.nih.gov/pubmed/16570500

49. Neag T, Olah NK, Hanganu D, Benedec D, Pripon FF, et al. The anemonin content of four different Ranunculus species. Pakistan J Pharmaceutical Sci. 2018; 31: 2027-2032.

PubMed: https://www.ncbi.nlm.nih.gov/pubmed/30393208

50. Mabona U, Viljoen A, Shikanga E, Marston A, Van Vuuren S. Antimicrobial activity of southern African medicinal plants with dermatological relevance: from an ethnopharmacological screening approach, to combination studies and the isolation of a bioactive compound. J Ethnopharmacol. 2013; 148: 45-55.

PubMed: https://www.ncbi.nlm.nih.gov/pubmed/23545456

51. You Y, Kim K, Yoon HG, Lee KW, Lee J, et al. Chronic effect of ferulic acid from Pseudosasa japonica leaves on enhancing exercise activity in mice. Phytotherapy Res. 2010; 24: 1508-1513.

PubMed: https://pubs.acs.org/doi/abs/10.1021/jf010514x

52. Matos P, Figueirinha A, Ferreira I, Cruz MT, Batista MT. Acanthus mollis L. leaves as source of anti-inflammatory and antioxidant phytoconstituents. Natural product research. 2019; 33: 1824-1827. PubMed: https://www.ncbi.nlm.nih.gov/pubmed/29417845

53. Santos-Rebelo A, Garcia C, Eleuterio C, Bastos A, Castro Coelho S, et al. Development of Parvifloron D-Loaded Smart Nanoparticles to Target Pancreatic Cancer. Pharmaceutics. 2018; 10: 216. PubMed: https://www.ncbi.nlm.nih.gov/pmc/articles/PMC6321128/

54. Feng X, Wang X, Liu Y, Di X. Linarin inhibits the acetylcholinesterase activity in-vitro and ex-vivo. Iranian journal of pharmaceutical research: IJPR. 2015; 14: 949.

PubMed: https://www.ncbi.nlm.nih.gov/pmc/articles/PMC4518125/

55. Saini NK, Singha M. Anti-inflammatory, analgesic and antipyretic activity of methanolic Tecomaria capensis leaves extract. Asian Pac J Trop Biomed. 2012; 2: 870-874.

PubMed: https://www.ncbi.nlm.nih.gov/pmc/articles/PMC3609241/

56. Aboutabl EA, Hashem FA, Sleem AA, Maamoon AA. Flavonoids, antiinflammatory activity and cytotoxicity of Macfadyena unguis-cati L. Afr J Tradit Complement Altern Med. 2008; 5: 18-26.

PubMed: https://www.ncbi.nlm.nih.gov/pmc/articles/PMC2816596/

57. Park SH, Jang S, Lee SW, Park SD, Sung YY, et al. Akebia quinata Decaisne aqueous extract acts as a novel anti-fatigue agent in mice exposed to chronic restraint stress. J Ethnopharmacol. 2018; 222 270-279.

PubMed: https://www.ncbi.nlm.nih.gov/pubmed/29630998

58. Guesmi F, Hmed MB, Prasad S, Tyagi AK, Landoulsi A. In vivo pathogenesis of colon carcinoma and its suppression by hydrophilic fractions of Clematis flammula via activation of TRAIL death machinery (DRs) expression. Biomedicine \& Pharmacotherapy. 2019; 109: 2182-2191.

59. Chu Z, Wang H, Ni T, Tao L, Xiang L, et al. 28-Hydroxy-3-oxoolean-12en-29-oic Acid, a Triterpene Acid from Celastrus Orbiculatus Extract, Inhibits the Migration and Invasion of Human Gastric Cancer Cells In Vitro. Molecules. 2019; 24: 3513.

PubMed: https://www.ncbi.nlm.nih.gov/pubmed/31569766

60. Habtemariam S. The therapeutic potential of Berberis darwinii stembark: quantification of berberine and in vitro evidence for Alzheimer's disease therapy. Nat Prod Commun. 2011; 6: 1089-1090.

PubMed: https://www.ncbi.nlm.nih.gov/pubmed/21922905

61. Topolska J, Kostecka-Gugała A, Ostachowicz B, Latowski D. Selected metal content and antioxidant capacity of Sambucus nigra flowers from the urban areas versus soil parameters and traffic intensity. Environ Sci Pollut Res Int. 2020; 27: 668-677.

PubMed: https://www.ncbi.nlm.nih.gov/pubmed/31808083

62. Packer J, Naz T, Harrington D, Jamie JF, Vemulpad SR. Antimicrobial activity of customary medicinal plants of the Yaegl Aboriginal community of northern New South Wales, Australia: a preliminary study. BMC Res Notes. 2015; 8: 276

PubMed: https://www.ncbi.nlm.nih.gov/pubmed/26122212

63. Kim HS, Jang JM, Yun SY, Zhou D, Piao Y, et al. Effect of Robinia pseudoacacia Leaf Extract on Interleukin-1ß-mediated Tumor Angiogenesis. In Vivo. 2019; 33: 1901-1910.

PubMed: https://www.ncbi.nlm.nih.gov/pubmed/31662518

64. Nawwar M, Swilam N, Hashim A, Al-Abd A, Abdel-Naim A, etal. Cytotoxic isoferulic acidamide from Myricaria germanica (Tamaricaceae). Plant Signal Behav. 2013; 8: e22642.

PubMed: https://www.ncbi.nlm.nih.gov/pmc/articles/PMC3745567/ 
65. Patova OA, Smirnov VV, Golovchenko VV, Vityazev FV, Shashkov AS, et al. Structural, rheological and antioxidant properties of pectins from Equisetum arvense L. and Equisetum sylvaticum L. Carbohydrate Polymers. 2019; 209: 239-249.

66. Calderon-Montano JM, Martinez-Sanchez SM, Burgos-Moron E, Guillen-Mancina E, Jimenez-Alonso JJ, et al. Screening for selective anticancer activity of plants from Grazalema Natural Park, Spain. Nat Prod Res. 2019; 33: 3454345-8.

PubMed: https://www.ncbi.nlm.nih.gov/pubmed/29842791

67. Pires AS, Rigueiras PO, Dohms SM, Porto WF, Franco OL. Structure-guided identification of antimicrobial peptides in the spathe transcriptome of the non-model plant, arum lily (Zantedeschia aethiopica). Chem Biol Drug Design. 2019; 93: 1265-1275.

68. Radulovic N, Denic M, Stojanovic-Radic Z. Antimicrobial phenolic abietane diterpene from Lycopus europaeus L.(Lamiaceae). Bioorg Med Chem Lett. 2010; 20: 4988-4891.

PubMed: https://www.ncbi.nlm.nih.gov/pubmed/20674349

69. Chen X, Li T, Qing D, Chen J, Zhang Q, et al. Structural characterization and osteogenic bioactivities of a novel Humulus lupulus polysaccharide. Food Function. 2020; 11: 1165-1175.

PubMed: https://www.ncbi.nlm.nih.gov/pubmed/31872841

70. Bournine L, Bensalem S, Wauters JN, Iguer-Ouada M, MaizaBenabdesselam $F$, et al.. Identification and quantification of the main active anticancer alkaloids from the root of Glaucium flavum. Int J Mol Sci. 2013; 14: 23533-23544.

PubMed: https://www.ncbi.nlm.nih.gov/pmc/articles/PMC3876061/

71. Basic M, Elgner F, Bender D, Sabino C, Herrlein ML, et al. A synthetic derivative of houttuynoid B prevents cell entry of Zika virus. Antiviral Res. 2019; 172: 104644.

PubMed: https://www.ncbi.nlm.nih.gov/pubmed/31697958

72. Akhtar M, Shaukat A, Zahoor A, Chen Y, Wang Y, et al.. Antiinflammatory effects of Hederacoside-C on Staphylococcus aureus induced inflammation via TLRs and their downstream signal pathway in vivo and in vitro. Microbial Pathogenesis. 2019; 137: 103767. PubMed: https://www.ncbi.nlm.nih.gov/pubmed/31580956

73. García ME, Borioni JL, Cavallaro V, Puiatti M, Pierini AB, et al. Solanocapsine derivatives as potential inhibitors of acetylcholinesterase: Synthesis, molecular docking and biological studies. Steroids. 2015; 104: 95-110.

PubMed: https://www.ncbi.nlm.nih.gov/pubmed/26362598

74. Dougnon G, Ito M. Sedative effects of the essential oil from the leaves of Lantana camara occurring in the Republic of Benin via inhalation in mice. J Nat Med. 2020; 74: 159-169.

PubMed: https://www.ncbi.nlm.nih.gov/pubmed/31446559

75. Hsu CL, Fang SC, Yen GC. Anti-inflammatory effects of phenolic compounds isolated from the flowers of Nymphaea mexicana Zucc. Food \& function. 2013; 4: 1216-1222.

PubMed: https://www.ncbi.nlm.nih.gov/pubmed/23727892

76. Ticona LA, SAnchez AR, GonzAles OO, Domenech MO. Antimicrobial compounds isolated from Tropaeolum tuberosum. Natural Product Research. 2020: 1-5.

PubMed: https://www.ncbi.nlm.nih.gov/pubmed/31913056

77. Luis A, Breitenfeld L, Ferreira S, Duarte AP, Domingues F. Antimicrobial, antibiofilm and cytotoxic activities of Hakea sericea Schrader extracts. Pharmacognosy magazine. 2014; 10 : S6.

PubMed: https://www.ncbi.nlm.nih.gov/pubmed/24914310

78. Yesilada E, Kupeli E. Clematis vitalba L. aerial part exhibits potent antiinflammatory, antinociceptive and antipyretic effects. J Ethnopharmacol. 2007; 110: 504-515.

79. Amabeoku GJ, Green I, Kabatende J. Anticonvulsant activity of Cotyledon orbiculata L.(Crassulaceae) leaf extract in mice. J Ethnopharmacol. 2007; 112: 101-107.

PubMed: https://pubmed.ncbi.nlm.nih.gov/17398051

80. Neuman MG, Jia AY, Steenkamp V. Senecio latifolius induces in vitro hepatocytotoxicity in a human cell line. Canadian journal of physiology and pharmacology. 2007; 85: 1063-1075.

81. dos Santos Alves CF, Bonez PC, de Souza MD, da Cruz RC, Boligon AA, et al. Antimicrobial, antitrypanosomal and antibiofilm activity of Equisetum hyemale. Microbial pathogenesis. 2016; 101: 119-125. PubMed: https://www.ncbi.nlm.nih.gov/pubmed/27856271

82. Schmidt M, Skaf J, Gavril G, Polednik C, Roller J, et al. The influence of Osmunda regalis root extract on head and neck cancer cell proliferation, invasion and gene expression. BMC Complement Altern Med. 2017; 17: 518.

PubMed: https://www.ncbi.nlm.nih.gov/pmc/articles/PMC5716017/

83. Tian G, Chen J, Luo Y, Yang J, Gao T, Shi J. Ethanol extract of Ligustrum lucidum Ait. leaves suppressed hepatocellular carcinoma in vitro and in vivo. Cancer Cell Int. 2019; 19: 246.

PubMed: https://www.ncbi.nlm.nih.gov/pubmed/31572063

84. Saddiqe Z, Maimoona A, Abbas G, Naeem I, Shahzad M. Pharmacological screening of Hypericum androsaemum extracts for antioxidant, anti-lipid peroxidation, antiglycation and cytotoxicity activity. Pakistan J Pharmaceut Sci. 2016; 29.

85. Tiana C, Yang C, Zhang D, Han L, Liu Y, et al. Antibacterial and antioxidant properties of various solvents extracts of Abutilon theophrasti Medic. leaves. Pak J Pharmaceut Sci. 2017; 30.

PubMed: https://www.ncbi.nlm.nih.gov/pubmed/28653920

86. Ling B, Xiao S, Yang J, Wei Y, Sakharkar MK, et al. Probing the Antitumor Mechanism of Solanum nigrum L. Aqueous Extract against Human Breast Cancer MCF7 Cells. Bioengineering. 2019; 6: 112. PubMed: https://www.ncbi.nlm.nih.gov/pubmed/31835887

87. Aghajanyan A, Nikoyan A, Trchounian A. Biochemical activity and hypoglycemic effects of Rumex obtusifolius $L$. seeds used in Armenian traditional medicine. BioMed Res Int. 2018; 2018.

88. Boniface PK, Verma S, Shukla A, Cheema HS, Srivastava SK, et al. Bioactivity-guided isolation of antiplasmodial constituents from Conyza sumatrensis (Retz.) EH Walker. Parasitol Int. 2015; 64: 118-123. PubMed: https://www.ncbi.nlm.nih.gov/pubmed/25449289

89. Genc Y, Dereli FT, Saracoglu I, Akkol EK. The inhibitory effects of isolated constituents from Plantago major subsp. major L. on collagenase, elastase and hyaluronidase enzymes: Potential wound healer. Saudi Pharmaceut J. 2020; 28: 101-106. PubMed: https://www.ncbi.nlm.nih.gov/pubmed/31920436

90. Rogozhin EA, Slezina MP, Slavokhotova AA, Istomina EA, Korostyleva TV, et al. A novel antifungal peptide from leaves of the weed Stellaria media L. Biochimie. 2015; 116: 125-132.

PubMed: https://www.ncbi.nlm.nih.gov/pubmed/26196691

91. Ilina T, Kashpur N, Granica S, Bazylko A, Shinkovenko I, et al. Phytochemical Profiles and In vitro Immunomodulatory Activity of Ethanolic Extracts from Galium aparine L. Plants. 2019; 8: 541.

PubMed: https://pubmed.ncbi.nlm.nih.gov/31775336

92. Aremu OO, Oyedeji AO, Oyedeji OO, Nkeh-Chungag BN, Rusike CR. In Vitro and In Vivo Antioxidant Properties of Taraxacum officinale in $\mathrm{N} \omega$-Nitro-I-Arginine Methyl Ester (L-NAME)-Induced Hypertensive Rats. Antioxidants. 2019; 8: 309.

PubMed: https://www.ncbi.nlm.nih.gov/pubmed/31443195

93. Jahan S, Azad T, Ayub A, Ullah A, Afsar T, et al. Ameliorating potency of Chenopodium album Linn. and vitamin $C$ against mercuric chlorideinduced oxidative stress in testes of Sprague Dawley rats. Environ Health Prevent Med. 2019; 24: 62. 
94. Parzonko A, Kiss AK. Caffeic acid derivatives isolated from Galinsoga parviflora herb protected human dermal fibroblasts from UVA-radiation. Phytomedicine. 2019; 57: 215-22.

PubMed: https://www.ncbi.nlm.nih.gov/pubmed/30785017

95. Di Sotto A, Di Giacomo S, Toniolo C, Nicoletti M, Mazzanti G. Sisymbrium Officinale (L.) Scop. and its polyphenolic fractions inhibit the mutagenicity of Tert-butylhydroperoxide in Escherichia Coli WP2uvrAR strain. Phytotherapy Res. 2016; 30: 829-834.

96. Di Napoli M, Varcamonti M, Basile A, Bruno M, Maggi F, et al. AntiPseudomonas aeruginosa activity of hemlock (Conium maculatum, Apiaceae) essential oil. Nat Prod Res. 2019; 33: 3436-3440.

97. Song SY, Hyun JE, Kang JH, Hwang CY. In vitro antibacterial activity of the manuka essential oil from Leptospermum scoparium combined with Tris-EDTA against Gram-negative bacterial isolates from dogs with otitis externa. Vet Dermatol. 2019.

98. Doukkali Z, Taghzouti K, Bouidida EH, Nadjmouddine M, Cherrah Y, et al. Evaluation of anxiolytic activity of methanolic extract of Urtica urens in a mice model. Behav Brain Funct. 2015; 11: 19.

PubMed: https://www.ncbi.nlm.nih.gov/pmc/articles/PMC4423131/

99. Farid O, Zeggwagh NA, Ouadi FE, Eddouks M. Mentha pulegium Aqueous Extract Exhibits Antidiabetic and Hepatoprotective Effects in Streptozotocin-induced Diabetic Rats. Endocrine, Metabolic \& Immune Disorders-Drug Targets (Formerly Current Drug Targets-Immune, Endocrine \& Metabolic Disorders). 2019; 19: 292-301.

PubMed: https://www.ncbi.nlm.nih.gov/pubmed/30289084

100. Bubueanu C, luksel R, Panteli M. Haemostatic activity of butanolic extracts of Lamium album and Lamium purpureum aerial parts. Acta Pharmaceutica. 2019; 69: 443-449.

PubMed: https://www.ncbi.nlm.nih.gov/pubmed/31259737

101. Ibrahim AM, Ghoname SI. Molluscicidal impacts of Anagallis arvensis aqueous extract on biological, hormonal, histological and molecular aspects of Biomphalaria alexandrina snails. Experimental Parasitology. 2018; 192: 36-41.

102. Fernandez-Martinez E, Jimenez-Santana M, Centeno-Alvarez M, Torres-Valencia JM, Shibayama M, et al. Hepatoprotective effects of nonpolar extracts from inflorescences of thistles Cirsium vulgare and Cirsium ehrenbergii on acute liver damage in rat. Pharmacognosy magazine. 2017; 13: S860.

PubMed: https://www.ncbi.nlm.nih.gov/pmc/articles/PMC5822512/

103. Sharifi-Rad J, Iriti M, Setzer WN, Sharifi-Rad M, Roointan A, et al Antiviral activity of Veronica persica Poir. on herpes virus infection. Cell Mol Biol. 2018; 64: 11-17.

PubMed: https://pubmed.ncbi.nlm.nih.gov/29981678

104. Li BY, Hu Y, Li J, Shi K, Shen YF, et al. Ursolic acid from Prunella vulgaris $L$. efficiently inhibits IHNV infection in vitro and in vivo. Virus Res. 2019; 273: 197741.

105. Torres-GonzAlez L, Cienfuegos-Pecina E, Perales-Quintana MM, Alarcon-Galvan G, Munoz-Espinosa LE, et al. Nephroprotective effect of sonchus oleraceus extract against kidney injury induced by ischemia-reperfusion in wistar rats. Oxidative medicine and cellular longevity. 2018; 2018: 9572803.

PubMed: https://www.ncbi.nlm.nih.gov/pubmed/29643981

106. Ayaz M, Ahmad I, Sadiq A, Ullah F, Ovais M, Khalil AT, Devkota HP. Persicaria hydropiper (L.) Delarbre: A review on traditional uses, bioactive chemical constituents and pharmacological and toxicological activities. Journal of Ethnopharmacology. 2019: 112516.

PubMed: https://www.ncbi.nlm.nih.gov/pubmed/31884037

107. Guz L, Adaszek L, Wawrzykowski J, Zietek J, Winiarczyk S. In vitro antioxidant and antibabesial activities of the extracts of Achillea millefolium. Polish journal of veterinary sciences. 2019: 369-376. PubMed: https://www.ncbi.nlm.nih.gov/pubmed/31269341
108. Mahdavi S, Amiradalat M, Babashpour M, Sheikhlooei H, Miransari $M$. The antioxidant, anticarcinogenic and antimicrobial properties of Verbascum thapsus L. Medicinal chemistry (Shariqah (United Arab Emirates)). 2019.

PubMed: https://pubmed.ncbi.nlm.nih.gov/31456524

109. Oszmianski J, Wojdyło A, Juszczyk P, Nowicka P. Roots and Leaf Extracts of Dipsacus fullonum L. and Their Biological Activities. Plants. 2020; 9: 78.

PubMed: https://www.ncbi.nlm.nih.gov/pubmed/31936189

110. Kim J, Jung KH, Ryu HW, Kim DY, Oh SR, et al. Apoptotic Effects of Xanthium strumarium via PI3K/AKT/mTOR Pathway in Hepatocellular Carcinoma. Evidence-Based Complementary and Alternative Medicine. 2019; 2019.

111. Shiomi N, editor. Advances in Bioremediation and Phytoremediation. BoD-Books on Demand; 2018.

112. Macci C, Peruzzi E, Doni S, lannelli R, Masciandaro G. Ornamental plants for micropollutant removal in wetland systems. Environ Sci Pollut Res. 2015; 22: 2406-2415.

PubMed: https://www.ncbi.nlm.nih.gov/pubmed/24798922

113. Augustynowicz J, Lukowicz K, Tokarz K, Płachno BJ. Potential for chromium (VI) bioremediation by the aquatic carnivorous plant Utricularia gibba L. (Lentibulariaceae). Environ Sci Pollut Res. 2015; 22: 9742-9748.

114. Cui X, Fang S, Yao Y, Li T, Ni Q, et al. Potential mechanisms of cadmium removal from aqueous solution by Canna indica derived biochar. Sci Total Environ. 2016; 562: 517-525.

PubMed: https://www.ncbi.nlm.nih.gov/pubmed/27107650

115. Wechtler L, Laval-Gilly P, Bianconi O, Walderdorff L, Bonnefoy A, et al. Trace metal uptake by native plants growing on a brownfield in France: zinc accumulation by Tussilago farfara L. Environ Sci Pollut Res . 2019; 26: 36055-36062

116. Maleva M, Garmash E, Chukina N, Malec P, Waloszek A, et al. Effect of the exogenous anthocyanin extract on key metabolic pathways and antioxidant status of Brazilian elodea (Egeria densa (Planch.) Casp.) exposed to cadmium and manganese. Ecotoxicol Environ Safety. 2018; 160: 197-206.

117. Marchand L, Lamy P, Bert V, Quintela-Sabaris C, Mench M. Potential of Ranunculus acris $L$. for biomonitoring trace element contamination of riverbank soils: photosystem II activity and phenotypic responses for two soil series. Environ Sci Pollut Res . 2016; 23: 3104-3119. PubMed: https://www.ncbi.nlm.nih.gov/pubmed/25956517

118. Pardo-Muras M, G Puig C, Pedrol N. Cytisus scoparius and Ulex europaeus Produce Volatile Organic Compounds with Powerful Synergistic Herbicidal Effects. Molecules. 2019; 24: 4539. PubMed: https://www.ncbi.nlm.nih.gov/pmc/articles/PMC6943486/

119. Salinitro M, Tassoni A, Casolari S, de Laurentiis F, Zappi A, et al. Heavy Metals Bioindication Potential of the Common Weeds Senecio vulgaris L., Polygonum aviculare L. and Poa annua L. Molecules. 2019; 24: 2813.

PubMed: https://www.ncbi.nlm.nih.gov/pubmed/31374997

120. Guo X, Wang P, LiY, Zhong H, Li P, et al. Effect of copper on the removal of tetracycline from water by Myriophyllum aquaticum: Performance and mechanisms. Bioresource Technol. 2019; 291: 121916. PubMed: https://www.ncbi.nlm.nih.gov/pubmed/31377514

121. Noori A, Zare Maivan H, Alaie E, Newman LA. Leucanthemum vulgare Lam. crude oil phytoremediation. Int J Phytoremediation. 2018; 20: 1292-1299.

PubMed: https://pubmed.ncbi.nlm.nih.gov/26121329

122. Yu X, Brown JM, Graham SE, Carbajal EM, Zuleta MC, Milla-Lewis SR. Detection of quantitative trait loci associated with drought 
tolerance in St. Augustinegrass. PloS one. 2019; 14.

PubMed: https://www.ncbi.nlm.nih.gov/pubmed/31671135

123. Alur HH, Pather SI, Mitra AK, Johnston TP. Evaluation of the gum from Hakea gibbosa as a sustained-release and mucoadhesive component in buccal tablets. Pharmaceutical development and technology. 1999; 4: 347-358.

PubMed: https://www.ncbi.nlm.nih.gov/pubmed/10434280

124. Keshari AK, Srivastava R, Singh P, Yadav VB, Nath G. Antioxidant and antibacterial activity of silver nanoparticles synthesized by Cestrum nocturnum. J Ayurveda Integrative Med. 2018.

125. Hussain N, Abbasi T, Abbasi SA. Generation of highly potent organic fertilizer from pernicious aquatic weed Salvinia molesta. Environ Sci Pollut Res Int. 2018; 25: 4989-5002.

PubMed: https://www.ncbi.nlm.nih.gov/pubmed/29209963

126. Balsamo RA, Willigen CV, Bauer AM, Farrant J. Drought tolerance of selected Eragrostis species correlates with leaf tensile properties. Ann Botany. 2006; 97: 985-991.

127. Zhou Y, Lambrides CJ, Kearns R, Ye C, Fukai S. Water use, water use efficiency and drought resistance among warm-season turfgrasses in shallow soil profiles. Functional plant biology. 2012; 39: 116-125. PubMed: https://www.publish.csiro.au/fp/FP11244
128. Baracho NC, Vicente BB, Arruda GD, Sanches BC, Brito JD. Study of acute hepatotoxicity of Equisetum arvense L. in rats. Acta Cirurgica Brasileira. 2009; 24: 449-453.

PubMed: https://www.ncbi.nlm.nih.gov/pubmed/20011829/

129. Slaughter RJ, Beasley DM, Lambie BS, Wilkins GT, Schep LJ. Poisonous plants in New Zealand: a review of those that are most commonly enquired about to the National Poisons Centre. N Z Med J. 2012; 125: 87-118.

PubMed: https://www.ncbi.nlm.nih.gov/pubmed/23321887

130. Bregnbak D, Menné T, Johansen JD. Airborne contact dermatitis caused by common ivy (Hedera helix L. ssp. helix). Contact Dermatitis. 2015; 72: 243-244.

PubMed: https://www.ncbi.nlm.nih.gov/pubmed/25630853

131. Pour BM, Sasidharan S. In vivo toxicity study of Lantana camara. Asian Pac J Trop Biomed. 2011; 1: 230-232.

PubMed: https://www.ncbi.nlm.nih.gov/pmc/articles/PMC3609184/

132. Botha CJ, Lessing D, Rösemann M, Van Wilpe E, Williams JH Analytical confirmation of Xanthium strumarium poisoning in cattle. J Vet Diagn Invest. 2014; 26: 640-645.

PubMed: https://pubmed.ncbi.nlm.nih.gov/25012081 